\title{
Classification of platelet concentrates (Platelet-Rich Plasma-PRP, Platelet-Rich Fibrin-PRF) for topical and infiltrative use in orthopedic and sports medicine: current consensus, clinical implications and perspectives
}

\author{
David M. Dohan Ehrenfest ${ }^{1}$ \\ Isabel Andia² \\ Matthias A. Zumstein ${ }^{3}$ \\ Chang-Qing Zhang ${ }^{4}$ \\ Nelson R. Pinto ${ }^{5}$ \\ Tomasz Bielecki ${ }^{6}$
}

1 LoB5 unit, Research Center for Biomineralization Disorders, School of Dentistry, Chonnam National University, Gwangju, South Korea and Department of Stomatology, Oral Surgery, and Dental and Maxillofacial Radiology, School of Dental Medicine, University of Geneva, Switzerland

2 Regenerative Medicine Laboratory, Cruces University Hospital, BioCruces Health Research Institute, Barakaldo, Spain

3 Shoulder, Elbow and Orthopedic Sports Medicine Unit, Department of Orthopaedics and Traumatology, Inselspital, University of Bern, Switzerland

4 Department of Orthopaedic Surgery, Shanghai Sixth People's Hospital Affiliated to Shanghai, Jiatong University, Shanghai, China

5 Department of Surgical and Prosthetic Implantology, Faculty of Odontology, University of the Andes (UANDES), Santiago, Chile

6 Department and Clinic of Orthopaedics, Medical University of Silesia, Sosnowiec, Poland

Corresponding author:

David M. Dohan Ehrenfest

School of Dental Medicine, University of Geneva 19 Barthelemy Menn, 1205 Geneva, Switzerland E-mail: LoB5@mac.com

\section{Summary}

Platelet concentrates for topical and infiltrative use - commonly termed Platetet-Rich Plasma (PRP) or Platelet-Rich Fibrin (PRF) - are used or tested as surgical adjuvants or regenerative medicine preparations in most medical fields, particularly in sports medicine and orthopaedic surgery. Even if these products offer interesting therapeutic perspectives, their clinical relevance is largely debated, as the literature on the topic is often confused and contradictory. The long history of these products was always associated with confusions, mostly related to the lack of consensual terminology, characterization and classification of the many products that were tested in the last $\mathbf{4 0}$ years. The current consensus is based on a simple classification system dividing the many products in $\mathbf{4}$ main families, based on their fibrin architecture and cell content: Pure Platelet-Rich Plasma (P-PRP), such as the PRGF-Endoret technique; Leukocyte- and Platelet-Rich Plasma (LPRP), such as Biomet GPS system; Pure PlateletRich Fibrin (P-PRF), such as Fibrinet; Leukocyte and Platelet-Rich Fibrin (L-PRF), such as IntraSpin L-PRF. The 4 main families of products present different biological signatures and mechanisms, and obvious differences for clinical applications. This classification serves as a basis for further investigations of the effects of these products. Perspectives of evolutions of this classification and terminology are also discussed, particularly concerning the impact of the cell content, preservation and activation on these products in sports medicine and orthopaedics.

KEY WORDS: blood platelet, fibrin, growth factors, leukocytes, regenerative medicine, sports medicine.

\section{Introduction}

The development of platelet concentrates for surgical use, often termed under the general acronyms PRP (Platelet-Rich Plasma) or PRF (Platelet-Rich Fibrin), is an important current transversal field of research across many fundamental and clinical disciplines ${ }^{1}$. These products are often associated with the keywords "growth factors", "regenerative medicine", "stem cells" and other magic-sounding fashion words. When considering these products, like many others, it is important to ask 3 good questions:

1. What are platelet concentrates for topical and infiltrative use?

2. Why do we use them exactly?

3. What are the results after 30 years of use?

Platelet concentrates for topical and infiltrative use are first of all blood extracts obtained after various processing of a whole blood sample, mostly through centrifugation ${ }^{1}$. The objective of the processing is to separate the blood components in order to discard elements considered as not usable (mostly the red blood cells, heavy and easily separated) and to gather and concentrate the elements that may be use for therapeutic applications (fibrinogen/fibrin, 
platelets, growth factors, leukocytes and other forms of circulating cells, in solution in liquid plasma) ${ }^{2}$. In short, all these products, whatever the technique used, are extracts of the blood circulating tissue. They are tissues themselves, and not pharmaceutical preparations.

These preparations are used on a surgical or wounded site in order to stimulate, improve and accelerate healing $^{3}$. In all wounds, the coagulation of blood to form a fibrin/platelet clot and matrix is the initial step of the natural healing process ${ }^{4}$. The use of platelet concentrates was designed to reinforce this natural process, like the fibrin glues used since more than 40 years as surgical adjuvants to improve healing ${ }^{5}$. With time, this concept of optimization of healing evolved to a more sophisticated concept of tissue regeneration promoted by the growth factors and the cells contained in these preparations: initially considered as surgical adjuvants, the PRP/PRF became the promoted glorious instruments of new regenerative medicine strategies ${ }^{1}$.

Based on the international scientific literature on the topic and the evolution of the clinical trends, it is difficult to state which products are really useful ${ }^{6,7}$. Many authors noticed that the published experimental results are difficult to sort and interpret, the clinical results are mixed or at least controversial and finally the relevance of use is debatable, considering the literature, the general feedback of experience and practical considerations (such as the heavy cost of most of these techniques). The reason of this regrettable result was highlighted in many recent debates and conferences ${ }^{8}$ :

- many different techniques for the production of platelet concentrates for surgical use are available (commercial marketed systems, or custommade systems developed for the need of some experimental studies) leading to very different final preparations;

- there was no proper terminology to classify and describe the many different variations of platelet concentrates:

- there are many confusions between the techniques and a lack of accurate characterization of the tested products in most articles on the topic, leading to a huge literature of thousands of articles constituting a "blind library of knowledge"2.

The answers to the 3 initial questions illustrate very well the current situation in the field. The need for clarification, terminology, categorization or classification was highlighted several years ago, but this endeavor is still at its inception. To understand the heart of these techniques, it is mandatory to remember the real history of these techniques.

\section{Long history and confusions}

The history of these preparations is often wrongly associated with the first article of Marx et al. in $1998^{9}$ about growth factors effects in Platelet-Rich Plasma (PRP). However, in reality, the history of these techniques started much earlier with the research works of
Matras ${ }^{10}$ about the fibrin glues used to improve skin wound healing in a rat model in 1970. These works lead to the fibrin glues still in use nowadays (such as the Tisseel from Baxter, Deerfield, IL, USA) ${ }^{5}$. In these technologies, the fibrin matrix is the main blood constituent (with a few other associated matrix proteins) what is used to stimulate healing, and it is therefore a bit limited in comparison to the full range of blood constituents that could be used. However, the fibrin matrix being the final result of the reaction chains of the coagulation and the first matrix of healing, the use of such glues represented one of the first biological surgical adjuvants developed in the modern era ${ }^{5}$.

A few years later (1975-1979) ${ }^{11}$, several research works proposed an upgraded concept for the use of blood extracts, termed "platelet-fibrinogen-thrombin mixtures" or "gelatin platelet - gel foam". In this new concept, the fibrin glues were including a significant concentration of platelets within the final preparation. The idea was first to reinforce naturally the fibrin gel, and also to combine the healing properties of the platelets with those of the fibrin. This upgrade of the fibrin glues allowed to prepare more natural products, integrating more natural blood constituents as it should. These techniques were the first platelet-rich plasma gels in the sense that we know today. These new strategies insisted in the role of platelets within the fibrin gel, and offered excellent preliminary results in ophthalmology, neurosurgery and general surgery. This approach was confirmed under other names in the following years, such as "platelet-derived wound healing factors or formula-PDWHF", and was tested with success for the treatment of skin ulcers ${ }^{12}$, following the principles developed with the fibrin glues 15 years earlier by Matras.

These techniques continued to develop slowly until the articles of Whitman in $1997^{13}$ and particularly Marx et al. in $1998^{\circ}$. These articles are the starting point of the craze for these techniques in oral and maxillofacial surgery and to the concept of platelet growth factors for regenerative medicine. From this moment, the number of publications and system available on the market grew quickly, and creating the situation of mass confusion described previously, while the products themselves were not fundamentally and conceptually different from what was used in the previous studies ${ }^{11}$. All these products were then termed Platelet-Rich Plasma, PRP, without consideration of their content or architecture, and this lack of terminology lasted many years.

At the same moment, another form of platelet concentrates was developed in France and termed Platelet-Rich Fibrin (PRF) ${ }^{14}$, due to the strong fibrin gel polymerization of the preparation. This technique was so obviously different from other PRPs, that it was termed a "second-generation" platelet concentrate, while this expression is probably not adequate considering the long history of evolutions of the platelet concentrates ${ }^{14}$. It is now considered simply as another family of products among others ${ }^{2}$. This evolution of terminology was however very important, as it was the first time that a product was obviously 
Classification of platelet concentrates (Platelet-Rich Plasma-PRP, Platelet-Rich Fibrin-PRF) for topical and infiltrative use in orthopedic and sports medicine: current consensus, clinical implications and perspectives

different enough from the others to justify a completely different terminology.

The second most important evolution of terminology only appeared in the last years, when several authors, particularly the groups of Dohan Ehrenfest ${ }^{15,16}$, Everts ${ }^{17,18}$ and Bielecki ${ }^{19,20}$, pointed out that these platelet concentrates were also associated with various forms of circulating cells, particularly leukocytes. The need for better consideration of the cell population was advocated in several articles $2,21,22$, and is now one of the most important source of debates in the field, particularly in sports medicine ${ }^{23}$.

To consider the history of these products illustrates the fashions that guided the research works during years. It started with an interest for the sole fibrin matrix as healing material ${ }^{10}$, then the priority was given to the healing properties of platelets ${ }^{11}$, and finally to the impact of growth factors (circulating and from the platelets $)^{9}$ for tissue regeneration. Finally, the role of the circulating cells became the new Frontier ${ }^{24}$. Among all these elements, which one can be considered as the most important? Considering our general knowledge about coagulation and healing - and some good sense - it is nowadays considered that all these elements are important and should be combined properly to reach the best clinical results. ${ }^{8}$ Fibrin, platelets, growth factors slow release, leukocytes and other cells: all these components are the key active actors of the natural healing process, and combined together are forming a kind of engineered tissue extracted from the blood circulating tissue ${ }^{25}$. This complex combination is the key for optimal performances. For this reason, the Leukocyte-and Platelet-Rich Fibrin L-PRF clot was often described as an "optimized blood clot" that can be surgically handled and used 25 . This expression is actually true (more or less) for all well-engineered platelet concentrates products.

\section{Current general classification}

Following the debates about the contents and the role of the various components of these preparations, a first classification was proposed in $2009^{2}$ and is now widely cited as a milestone in the process of clarification of the terminology. This classification is actually very simple, and separated the products following at least 2 key parameters: the presence of a cell content (mostly leukocytes) and the fibrin architecture. This separation allowed to define 4 main families to regroup the products.

1. Pure Platelet-Rich Plasma (P-PRP) - or Leukocyte-Poor Platelet-Rich Plasma - products are preparations without leukocytes and with a lowdensity fibrin network after activation. Per definition, all the products of this family can be used as liquid solutions or in an activated gel form. It can therefore be injected (for example in sports medicine) or placed during gelling on a skin wound or suture (similar to the use of fibrin glues).

Many methods of preparation exist, particularly using cell separators (continuous flow plasmapheresis) from hematology laboratory as suggested by many authors, even if this method is much too heavy to be used frequently and easily in daily practice. One largely advertised method of P-PRP is known under the commercial name PRGF 26 [Plasma Rich in Growth Factors or Preparations Rich in Growth Factors or EndoRet, Biotechnology Institute BTI (dental implant company), Vitoria, Spain] and was tested in many clinical situations, particularly in sports medicine. Significant issues of the technique are its lack of ergonomics and the need for approximate pipetting steps during the preparation ${ }^{2}$. The literature on this technique remains very difficult to evaluate, as most articles were produced by the company promoting it ${ }^{21}$. Another technique of P-PRP was widely promoted for skin ulcers and is known under the commercial name Vivostat PRF (Platelet-Rich Fibrin, Vivostat $\mathrm{A} / \mathrm{S}$, Alleroed, Denmark), what can be a source of confusion as this technique is not a PRF following the terminology, but clearly a P-PRP product ${ }^{2}$.

2. Leukocyte-and Platelet-Rich Plasma (L-PRP) products are preparations with leukocytes and with a low-density fibrin network after activation. Per definition, like the P-PRP, all the products of this familycan be used as liquid solutions or in an activated gel form ${ }^{17}$. It can therefore be injected (for example in sports medicine) or placed during gelling on a skin wound or suture (similar to the use of fibrin glues). It is in this family that the largest number of commercial or experimental systems exists with many interesting results in general surgery ${ }^{27}$, orthopaedic and sports medicine ${ }^{28}$. Particularly many automated protocols have been developed in the last years, requiring the use of specific kits that allow minimum handling of the blood samples and maximum standardization of the preparations, for example Harvest Smart-PreP (Harvest Technologies, Plymouth, MA, USA) and Biomet GPS III (Biomet Inc., Warsaw, IN, USA). Other kits with more handling also exist, such as Plateltex (Prague, Czech Republic) or Regen PRP (RegenLab, Le Mont-sur-Lausanne, Switzerland) ${ }^{2}$.

3. Pure Platelet-Rich Fibrin (P-PRF) - or LeukocytePoor Platelet-Rich Fibrin - are preparations without leukocytes and with a high-density fibrin network. Per definition, these products only exist in a strongly activated gel form, and cannot be injected or used like traditional fibrin glues. However, because of their strong fibrin matrix, they can be handled like a real solid material for other applications. There is only one product in this family, commercially known as Fibrinet PRFM (PlateletRich Fibrin Matrix, Cascade Medical, Wayne, NJ, USA, also marketed for orthopedic applications by Vertical Spine, Marconi Road Wall, NJ, USA). The main inconvenient of this technique remains its cost and relative complexity in comparison to the other forms of PRF available, the L-PRF (Leukocyte- and Platelet-Rich Fibrin) ${ }^{2}$. 
4. Leukocyte- and Platelet-Rich Fibrin (L-PRF) products are preparations with leukocytes and with a high-density fibrin network ${ }^{25}$. Per definition, these products only exist in a strongly activated gel form, and cannot be injected or used like traditional fibrin glues. However, because of their strong fibrin matrix, they can be handled like a real solid material for other applications.

The technique was initially developed and evaluated as an open-access technique, based on the concept of one-step centrifugation of blood without anticoagulant and without blood activator ${ }^{14}$ : the preparation is completely natural, and this remains a key difference with all other families of products ${ }^{2}$. Nowadays, the only FDA-approved CE-marked system of L-PRF with certified materials is marketed under the name IntraSpin L-PRF (Intra-Lock Inc., Boca Raton, FL, USA) ${ }^{29-}$ ${ }^{31}$.The technique is very simple, quick, inexpensive and allows to produce large quantities of fibrin clots and membranes in a very short time, particularly using the Xpression preparation box ${ }^{32}$. This is currently the main technique in oral and maxillofacial surgery, particularly because the L-PRF membranes and clots are very easy to combine with current surgical techniques $^{6,7}$. Some applications of this technique were proposed with interesting results in sports medicine and orthopaedics ${ }^{33,34}$, but these applications remain still experimental as they require to find a way to use the clots in each specific surgical procedure (how to maintain the membranes/clots in adequate position), while PRPs families are often simply injected like a pharmaceutical preparation ${ }^{28}$.

This classification system was largely cited, advocated, and validated by a multi- disciplinary consensus conference published in $2012^{8}$. The POSEIDO (Periodontology, Oral Surgery, Esthetic and Implant Dentistry Organization) hold it as its guidelines for all publications on the topic in $2013^{30}$. This terminology and classification are now considered as a basis of consensus in many fields, particularly in oral and maxillofacial disciplines, but many other evolutions may be needed in the future, with more or less relevance depending on the clinical field.

\section{From terminology to biological mechanisms}

The 2 parameters selected to define the 4 families of products are obvious and logical, and there is a consensus on their importance. Any researcher handling these products can observe immediately the significant differences between these 4 families. These differences must be also highlighted and quantified through biological and clinical parameters.

Each family of products presents some major specificities, but each product individually has its own identity. A part of this identity was defined and investigated as its biological signature, in the sense of quantity and duration of the slow release of growth factors. Several studies $16,22,32,34$ tried to evaluate and compare the biological signature of these mate- rials. The in vitro behaviors of L-PRF membrane and P-PRP gel were compared ${ }^{22,34}$, through the evaluation of the slow release of growth factors and matrix molecules. These 2 families of gels were placed in culture medium during 7 days, and the slow releases of 3 key growth factors [Transforming Growth Factor $\beta 1$ (TGF $\beta 1$ ), Platelet-Derived Growth Factor AB (PDGF-AB), Vascular Endothelial Growth Factor (VEGF)] and 3 key coagulation and matrix proteins [Thrombospondin 1 (TSP1), Fibronectin, Vitronectin] were quantified at seven experimental times: 20 min, 1h, 4h, 24h (day 1), 72h (day 3), 120h (day 5) and $168 \mathrm{~h}$ (day 7 ). These studies revealed that the products presented 2 very different profiles: the LPRF membrane remained solid and intact after 7 days and released continuously a large quantity of growth factors, a significant part of it being produced by the cell population within the membrane ${ }^{16}$. On the contrary, the P-PRP gel released most of its growth factors in the first hours and completely dissolved in the medium after 3 days, even after a maximum artificial fibrin polymerization. These studies confirmed the previous works ${ }^{35}$ about the differences of the fibrin architecture between the PRF families (natural polymerization with intrinsic growth factors enmeshment) and the PRP gels families (artificial provoked polymerization with extrinsic growth factors enmeshment, leading to their immediate release and use/destruction) that served as the basis for the classification system.

These studies highlighted very different biological signatures and mechanisms between different families of products, but many other differences may be found within families themselves when considering particularly the variations of cell populations and preservation.

\section{From terminology to general clinical implications}

The classification of the products and the identification of their many differences also allowed to understand that each family of product has its own characteristics and specific clinical potential applications. With a general overview of the literature on the topic, it allows to reach some preliminary statements:

- the L-PRF family fits the needs of the applications in oral and maxillofacial ${ }^{6,7}$ surgery, as L-PRF clots and membranes present a volume and shape easy to combine with most surgical techniques, as filling and interposition healing biomaterial or as protection healing membrane. These membranes are also strong and offer a slow release of many growth factors during long periods. Finally, it is easy to prepare in large quantity and inexpensive, what makes it particularly adapted for daily clinical practice ${ }^{32}$

- PRF families in general are usable in other disciplines with interesting results, particularly for the treatment of skin wound ulcers ${ }^{3}$. However, these products only exist in a strongly polymerized acti- 
Classification of platelet concentrates (Platelet-Rich Plasma-PRP, Platelet-Rich Fibrin-PRF) for topical and infiltrative use in orthopedic and sports medicine: current consensus, clinical implications and perspectives

vated form: some applications were described in orthopedic and sports medicine ${ }^{33,34}$, but the PRF products cannot be used as injectable products in sports medicine for example.

- the various PRP families are not adapted (complicated, expensive, with mixed clinical relevance) for daily oral applications ${ }^{6,7}$, but are interesting substitutions to fibrin glues in most other surgeries, particularly to improve skin wound healing ${ }^{27}$. The use of gelling of the PRP on the surgical site makes it an adequate surgical adjuvants in many situations, even if the exact effects - in comparison to fibrin glues - remain largely debated ${ }^{27}$.

- the PRP solutions have also the advantage to be liquid before activation, and can therefore be used as injection in various sports medicine or orthopedic applications. In this strategy of regenerative medicine, the platelet suspensions are injected like other pharmaceutical preparations ${ }^{23}$. The results of this method remain however largely debated in the literature, probably because of the large quantity of different protocols 36,37 .

These first statements give a general overview of the current situation. If there is almost no more debates about which techniques to use in oral and maxillofacial surgery (the PRP fashion being largely abandoned nowadays due to its cost, complexity and lack of real interest, in comparison to the L-PRF technique), the situation is much more confused in other fields. There is particularly a very large debate in sports medicine on the selection of the adequate technique, particularly concerning the exact cell content of the injectable platelet suspensions ${ }^{23,36,37}$. Some groups advocated that the presence of leukocytes may be negative for the therapeutic outcome, due to a potential risk of stimulation of the inflammatory process after the injection in a wounded site ${ }^{26}$. On the contrary, other groups insisted on the need of some leukocyte population in the injectable $\mathrm{PRP}^{23}$, in order to increase the growth factors production, the release of anti-pain mediators ${ }^{24}$ and the natural antiinfectious activity ${ }^{38}$. In general, many leukocytes particularly lymphocytes - are playing a key function as regulation turntable of the healing and inflammatory process, and there is no serious reason (or reported published results) to discard them.

Leukocytes are not only inflammatory cells, as they also present anti-nociceptive effects through different chemokines, anti-inflammatory cytokines (IL-4, IL-10 and IL-13) and opioid peptides (b-endorphin, metenkephalin, and dynorphin-A), and can therefore promote a clinically relevant inhibition of pathological pain. During inflammation, these cytokines counteract the effects of the pro-inflammatory mediators generated naturally in the early stages of inflammation. The current unpublished consensus on this matter is that leukocytes are probably beneficial, but it depends which leukocytes (lymphocytes, monocytes, granulocytes), in which quantity and in which state (the centrifugation process can softly activate, pathologically stimulate inflammatory state, or destroy the white cells). This remains one of the most important point of discussion nowadays.

\section{Other classifications suggested in sports medi- cine}

The classification described previously is the only system covering all forms of platelet concentrates for surgical use. However, 2 other classification systems were proposed in the recent years, but are limited as they only refer to Platelet-Rich Plasma products and sports medicine applications.

1. Mishra et al..$^{23}$ proposed a classification only for sports medicine applications and taking into consideration the platelets and leukocytes concentrations. This classification creates 4 types of PRP, depending on the presence or absence of leukocytes and on the activation or not of the PRP. Following this proposal, type 1 PRP is a L-PRP solution, type 2 PRP is a L-PRP gel, type 3 PRP is PPRP solution, type 4 PRP is a P-PRP gel. This classification follows therefore the same idea than the general classification published in $2009^{2}$, but is more limited (PRP only) and less intuitive (types of PRPs are less obvious than clear terminology).

The only new parameter of this classification is the evaluation of the platelet concentration, type A PRP being 5-time or more the blood concentration of platelets, and type B PRP being less than 5 times the blood concentration of platelets. This last parameter is debatable, as the concept of platelet concentration was largely abandoned in the previous years for a logical reason: platelet concentration depends only on the volume of liquid serum used to keep the platelets in suspension. The quantity of serum varies a lot depending on the protocol and the expected application, and has no impact on the expected effect. The concept of absolute quantity of platelets would be more logical, even if most publications failed to detect a clear and reproducible impact of this parameter in the clinical outcomes ${ }^{39}$. From this standpoint the 5-time threshold has no consensual sense and justification.

2. Another system called PAW (Platelets, Activation, White cells $)^{40}$ was proposed to organize and compare results in the literature, and it insists on the platelet quantity (absolute number), the activation mode of the platelets and the presence of white cells. This system again is limited and only covers the PRP families, and is in fact very similar to the proposal of Mishra et al. ${ }^{23}$. Leukocytes and activation (liquid or gel) are already well isolated parameters ${ }^{2}$, and the question of the platelet quantity remains still a significant debate, as no publication was really able to define what would be an optimal platelet quantity, or even if the concept really exists with complex multi-components materials such as platelet concentrates ${ }^{39}$. 
Both proposals are interesting, but are not significantly evidence-based and do not really allow to upgrade the current terminology and classification as defined in $2009^{2}$.

\section{Perspectives of evolutions of the classification}

The 2009 terminology and classification ${ }^{2}$ are an important step, but remain probably incomplete considering the number of parameters involved in the characterization of such complex products. From a biological standpoint, the characterization of the presence of cells (such as leukocytes) is a critical step, but many other parameters should be considered, such $a^{2}$ : the platelet collection rate/quantity, the leukocyte collection rate/quantity, the detailed cell composition and the preservation (shape and stress level) of the cells during the collection and centrifugation. The activation of the cell content during or after the centrifugation is also important for the biology of these products. Other practical parameters should also be considered, as they impact directly the possibility to use these techniques in daily clinical practice, such as: the size of the centrifuge, the duration, cost and ergonomics of the preparation procedure, the final volume of product and its form (liquid, light gel or solid gel material). Finally, as it was clearly stated since the first classification article in $2009^{2}$, all these parameters have to be considered together. This is still far from being so obvious when observing the current literature, even if some improvement in the characterization of tested products can be observed.

The classification and terminology will evolve in the next years and it is expected that these evolutions will be found in the exact cell content of the L-PRP and L-PRF families ${ }^{24}$. Most publications about growth factors and platelet concentrations have shown the relative lack of significance of these parameters, due to the many inter-individual variations and the shortterm effects of these parameters ${ }^{39,41}$, platelets being activated and active during only a very short time period and growth factors being released, consumed locally or dissolved in the blood flow in the minutes or hours after their release. It is expected that the explanation of the mixed clinical results reported in the literature will be found in the cell population and activation of these products. Platelet concentrates for surgical use must be thought as the integration of all blood elements within a logical healing platform including the fibrin matrix, the platelets, the mediators and the cells all together to reach a clear and reproducible clinical result ${ }^{25}$.

Many cell types are present in these preparations. The exact leukocyte formula is an important parameter: Iymphocytes populations are very diverse and do not have at all the same impact than the monocytes and granulocytes. Moreover, many other cells - such as circulating stem cells - can be found in a platelet concentrate and shall not be neglected. Finally, it is still unclear how to improve significantly the current classification and terminology, but this is very much in these various aspects that evolutions may be found in the future. In the meanwhile, it is important for all authors in the field to describe accurately the products they are testing ${ }^{42}$, in order to do a real and significant contribution to the literature on this simple but difficult topic.

\section{Disclosure of interests}

The authors have no conflict of interest to report.

\section{Acknowledgement}

This work for the definition of international standards in implantable materials is supported by a grant from the National Research Foundation of Korea (NRF) funded by the Korean government- MEST (No. 20110030121) and by the LoB5 Foundation for Research, France.

\section{References}

1. Bielecki T, Dohan Ehrenfest DM. Platelet-rich plasma (PRP) and Platelet-Rich Fibrin (PRF): surgical adjuvants, preparations for in situ regenerative medicine and tools for tissue engineering. Curr Pharm Biotechnol. 2012;13:1121-1130.

2. Dohan Ehrenfest DM, Rasmusson L, Albrektsson T. Classification of platelet concentrates: from pure platelet-rich plasma (P-PRP) to leucocyte- and platelet-rich fibrin (L-PRF). Trends Biotechnol. 2009;27:158-167.

3. Cieslik-Bielecka A, Choukroun J, Odin G, Dohan Ehrenfest DM. L-PRP/L-PRF in esthetic plastic surgery, regenerative medicine of the skin and chronic wounds. Curr Pharm Biotechnol. 2012;13:1266-1277.

4. Clark RA. Fibrin and wound healing. Ann N Y Acad Sci 2001;936:355-367.

5. Gibble JW, Ness PM. Fibrin glue: the perfect operative sealant? Transfusion 1990;30:741-747.

6. Del Corso M, Vervelle A, Simonpieri A, et al. Current knowledge and perspectives for the use of platelet-rich plasma (PRP) and platelet-rich fibrin (PRF) in oral and maxillofacial surgery part 1: Periodontal and dentoalveolar surgery. Curr Pharm Biotechnol. 2012;13:1207-1230.

7. Simonpieri A, Del Corso M, Vervelle A, et al. Current knowledge and perspectives for the use of platelet-rich plasma (PRP) and platelet-rich fibrin (PRF) in oral and maxillofacial surgery part 2: Bone graft, implant and reconstructive surgery. Curr Pharm Biotechnol. 2012;13:1231-1256.

8. Dohan Ehrenfest DM, Bielecki T, Mishra A, et al. In search of a consensus terminology in the field of platelet concentrates for surgical use: platelet-rich plasma (PRP), platelet-rich fibrin (PRF), fibrin gel polymerization and leukocytes. Curr Pharm Biotechnol. 2012;13:1131-1137.

9. Marx RE, Carlson ER, Eichstaedt RM, Schimmele SR, Strauss JE, Georgeff KR. Platelet- rich plasma: Growth factor enhancement for bone grafts. Oral Surg Oral Med Oral Pathol Oral Radiol Endod. 1998;85:638-646.

10. Matras H. Die Wirkungen verschiedener Fibrinpräparate auf Kontinuitat-strennungen der Rattenhaut. Osterr Z Stomatol. 1970;67:338-359.

11. Rosenthal AR, Egbert PR, Harbury C, Hopkins JL, Rubenstein E. Use of platelet- fibrinogen-thrombin mixture to seal experi- 
Classification of platelet concentrates (Platelet-Rich Plasma-PRP, Platelet-Rich Fibrin-PRF) for topical and infiltrative use in orthopedic and sports medicine: current consensus, clinical implications and perspectives

mental penetrating corneal wounds. Albrecht Von Graefes Arch Klin Exp Ophthalmol. 1978;207:111-115.

12. Knighton DR, Ciresi KF, Fiegel VD, Austin LL, Butler EL. Classification and treatment of chronic nonhealing wounds. Successful treatment with autologous platelet-derived wound healing factors (PDWHF). Ann Surg. 1986;204:322-330.

13. Whitman DH, Berry RL, Green DM. Platelet gel: an autologous alternative to fibrin glue with applications in oral and maxillofacial surgery. J Oral Maxillofac Surg. 1997;55:1294-1299.

14. Dohan DM, Choukroun J, Diss A, et al. Platelet-rich fibrin (PRF): a second-generation platelet concentrate. Part I: technological concepts and evolution. Oral Surg Oral Med Oral Pathol Oral Radiol Endod. 2006; 101:e37-44.

15. Dohan DM, Choukroun J, Diss A, et al. Platelet-rich fibrin (PRF): a second-generation platelet concentrate. Part III: leucocyte activation: a new feature for platelet concentrates? Oral Surg Oral Med Oral Pathol Oral Radiol Endod. 2006;101:e51-55.

16. Dohan Ehrenfest DM, de Peppo GM, Doglioli P, Sammartino G. Slow release of growth factors and thrombospondin-1 in Choukroun's platelet-rich fibrin (PRF): a gold standard to achieve for all surgical platelet concentrates technologies. Growth Factors. 2009;27:63-69.

17. Everts PA, Hoffmann J, Weibrich G, et al. Differences in platelet growth factor release and leucocyte kinetics during autologous platelet gel formation. Transfus Med. 2006;16:363368.

18. Everts PA, van Zundert A, Schonberger JP, Devilee RJ, Knape JT. What do we use: platelet-rich plasma or plateletleukocyte gel? J Biomed Mater Res A. 2008;85:1135-1136.

19. Cieslik-Bielecka A, Gazdzik TS, Bielecki TM, Cieslik T. Why the platelet-rich gel has antimicrobial activity? Oral Surg Oral Med Oral Pathol Oral Radiol Endod. 2007;103:303-305; author reply 305-306.

20. Cieslik-Bielecka A, Bielecki T, Gazdzik TS, Arendt J, Krol W, Szczepanski T. Autologous platelets and leukocytes can improve healing of infected high-energy soft tissue injury. Transfus Apher Sci. 2009;41:9-12.

21. Dohan Ehrenfest DM, Bielecki T, Del Corso M, Inchingolo F, Sammartino G. Shedding light in the controversial terminology for platelet-rich products: platelet-rich plasma (PRP), plateletrich fibrin (PRF), platelet-leukocyte gel (PLG), preparation rich in growth factors (PRGF), classification and commercialism. $J$ Biomed Mater Res A. 2010;95:1280-1282.

22. Dohan Ehrenfest DM, Bielecki T, Jimbo R, et al. Do the fibrin architecture and leukocyte content influence the growth factor release of platelet concentrates? An evidence-based answer comparing a pure platelet-rich plasma (P-PRP) gel and a leukocyte- and platelet-rich fibrin (L-PRF). Curr Pharm Biotechnol. 2012;13:1145-1152.

23. Mishra A, Harmon K, Woodall J, Vieira A. Sports medicine applications of platelet rich plasma. Curr Pharm Biotechnol. 2012;13:1185-1195.

24. Bielecki T, Dohan Ehrenfest DM, Everts PA, Wiczkowski A The role of leukocytes from L-PRP/L-PRF in wound healing and immune defense: new perspectives. Curr Pharm Biotechnol. 2012;13:1153-1162.

25. Dohan Ehrenfest DM, Del Corso M, Diss A, Mouhyi J, Charrier JB. Three-dimensional architecture and cell composition of a Choukroun's platelet-rich fibrin clot and membrane. J Periodontol. 2010;81:546-555.

26. Anitua E, Sanchez M, Orive G, Andia I. The potential impact of the preparation rich in growth factors (PRGF) in different medical fields. Biomaterials. 2007;28:4551-4560.
27. Everts PA, Hoogbergen MM, Weber TA, Devilee RJ, van Monftort G, de Hingh IH. Is the use of autologous platelet-rich plasma gels in gynecologic, cardiac, and general, reconstructive surgery beneficial? Curr Pharm Biotechnol. 2012;13:11631172.

28. Yuan T, Guo SC, Han P, Zhang CQ, Zeng BF. Applications of leukocyte- and platelet- rich plasma (L-PRP) in trauma surgery. Curr Pharm Biotechnol. 2012;13:1173-1184.

29. Del Corso M, Dohan Ehrenfest DM. Immediate implantation and peri-implant Natural Bone Regeneration (NBR) in the severely resorbed posterior mandible using Leukocyte- and Platelet-Rich Fibrin (L-PRF): a 4-year follow-up. POSEIDO. 2013;1:109-116.

30. Dohan Ehrenfest DM, Sammartino G, Shibli JA, Wang HL, Zou DR, Bernard JP. Guidelines for the publication of articles related to platelet concentrates (Platelet-Rich Plasma - PRP, or Platelet-Rich Fibrin - PRF): the international classification of the POSEIDO. POSEIDO. 2013;1:17-27.

31. Toeroek R, Dohan Ehrenfest DM. The concept of Screw-Guided Bone Regeneration (S- GBR). Part 2: S-GBR in the severely resorbed preimplant posterior mandible using bone xenograft and Leukocyte- and Platelet-Rich Fibrin (L-PRF): a 5-year follow-up. POSEIDO 2013;1:85-92.

32. Dohan Ehrenfest DM. How to optimize the preparation of leukocyte- and platelet-rich fibrin (L-PRF, Choukroun's technique) clots and membranes: introducing the PRF Box. Oral Surg Oral Med Oral Pathol Oral Radiol Endod. 2010;110:275278; author reply 278-280.

33. Zumstein MA, Berger S, Schober M, et al. Leukocyte- and platelet-rich fibrin (L-PRF) for long-term delivery of growth factor in rotator cuff repair: review, preliminary results and future directions. Curr Pharm Biotechnol. 2012;13:1196-1206.

34. Zumstein MA, Bielecki T, Dohan Ehrenfest DM. The Future of Platelet Concentrates in Sports Medicine: Platelet-Rich Plasma, Platelet-Rich Fibrin, and the Impact of Scaffolds and Cells on the Long-term Delivery of Growth Factors. Operative Techniques in Sports Medicine. 2011;19:190-197.

35. Dohan DM, Choukroun J, Diss A, et al. Platelet-rich fibrin (PRF): a second-generation platelet concentrate. Part II: platelet-related biologic features. Oral Surg Oral Med Oral Pathol Oral Radiol Endod. 2006;101:e45-50.

36. Andia I, Maffulli N. Platelet-rich plasma for muscle injury and tendinopathy. Sports Med Arthrosc. 2013;21:191-198.

37. Andia I, Maffulli N. Platelet-rich plasma for managing pain and inflammation in osteoarthritis. Nat Rev Rheumatol. 2013;9: 721-730.

38. Moojen DJ, Everts PA, Schure RM, et al. Antimicrobial activity of platelet-leukocyte gel against Staphylococcus aureus. J Orthop Res. 2008;26:404-410.

39. Weibrich G, Hansen T, Kleis W, Buch R, Hitzler WE. Effect of platelet concentration in platelet-rich plasma on peri-implant bone regeneration. Bone. 2004;34:665-671.

40. DeLong JM, Russell RP, Mazzocca AD. Platelet-rich plasma: the PAW classification system. Arthroscopy. 2012;28:9981009.

41. Weibrich G, Kleis WK, Kunz-Kostomanolakis M, Loos AH, Wagner W. Correlation of platelet concentration in plateletrich plasma to the extraction method, age, sex, and platelet count of the donor. Int J Oral Maxillofac Implants 2001;16: 693-699.

42. Padulo J, Oliva F, Frizziero A, Maffulli N. Muscle, Ligaments and Tendons Journal. Basic principles and recommendations in clinical and field science research. MLTJ. 2013;4:250-252. 